\section{Dealing with West Nile Virus: Evaluate, re-evaluate, respond}

Rosmarie Kelly, $\mathrm{PhD}^{1}$

${ }^{1}$ Public Health Epidemiologist, Environment Health Section, Georgia Department of Public Health, Atlanta, GA

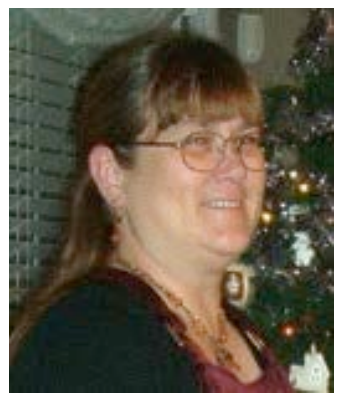

West Nile virus (WNV) is a mosquito-borne viral pathogen that was introduced into the United States in 1999. Within four years following its initial detection in New York, WNV was found in states from the East to the West coasts as well as in Mexico and Canada.

WNV, an arbovirus, is maintained in birds; its presence in Georgia was first confirmed in July 2001 when an American crow from Lowndes County tested positive for the virus. The virus occasionally infects humans who are bitten by mosquitoes that have been feeding on birds. Most people (approximately 80\%) infected with WNV do not develop symptoms. About one in five infected people experiences a relatively mild illness, often termed "West Nile Fever", characterized by fever, headache, muscle weakness or myalgia, arthralgia, and sometimes rash. Less than one percent of persons infected with WNV develop neurologic illness ("West Nile Neurologic Disease" or WNND) in the form of meningitis, encephalitis, or possibly acute flaccid paralysis. Approximately three to fifteen percent of WNND cases are fatal. An increased risk of WNND is associated with increasing age and the presence of underlying medical conditions. Recently, in Georgia, there have been human WNV cases each year, ranging from a low of 6 cases in 2001 to a high of 117 cases in 2012.

Integrated management practices for mosquito control include education, surveillance, source reduction, larviciding, and adulticiding as the means to reduce mosquito populations below a threshold amount. Reminding the public at the start of mosquito season that wearing repellent reduces the risk of $\mathrm{WNV}$ is essential, as is reminding people of their role in reducing mosquito populations by eliminating mosquito-breeding areas in their yards and in their neighborhoods. This message should include "tip and toss"; at least once a week correctly dispose of debris that can contain water; dump out anything that can hold water. This includes wading pools, pots, buckets, saucers under plants, toys, leaves, tarps, and plastic bags. Applying a larvicide to catch basins and other locations starting in March or April will help reduce populations of Culex spp mosquitoes, the principal vectors of WNV. Two larvicidal products are available to consumers, Mosquito Dunks ${ }^{\circledR}$ (Bacillus thuringiensis serotype israelenis) and Mosquito Torpedoes ${ }^{\circledR}$ (methoprene). It is necessary to read and follow label directions when using either of these products.
Application of larvicides and education by public health officials in response to positive birds or increased numbers of vectors has the effect of minimizing the risk of human disease in areas where the virus is actively circulating. When adult mosquito populations begin to rise, adulticiding, either area-wide or a more localized barrier spray, should be considered to reduce mosquito populations. If a municipal mosquito control program does not exist in their area, residents may choose to contract with a commercial mosquito control company or to apply a barrier spray in their own yards. Additional information concerning mosquito control can be found on the Georgia Mosquito Control website at www.GAmosquito.org.

Information is the key to reducing the risk of WNV transmission. Data obtained from mosquito surveillance efforts leads to responsible and informed decisions about mosquito control, as well as public education about elimination of mosquito breeding habitats and prevention of mosquito bites. For this disease, communication between agencies and the public is an essential part of the risk reduction effort.

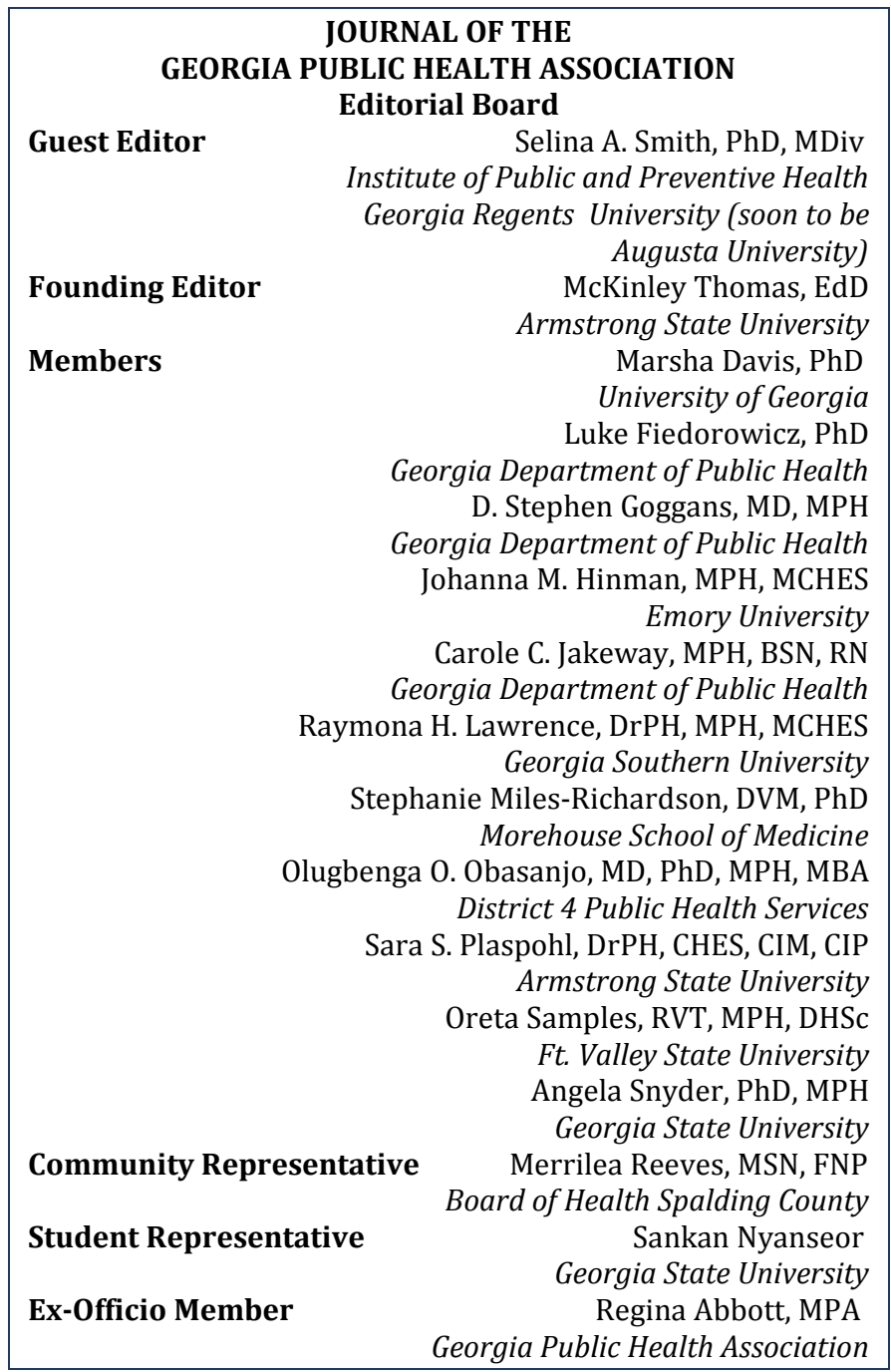

